\title{
Hybrid Neural Network Model based on Multi-Layer Perceptron and Adaptive Resonance Theory ${ }^{1}$
}

\author{
Andrey Gavrilov, Young.-Koo Lee, Sungyoung Lee \\ Department of Computer Engineering, Kyung Hee University, 1, Soechen-ri, Giheung-eop, \\ Yongin-shi, Gyeonggi-do, 449-701, Korea \\ Avg1952@rambler.ru, yklee@khu.ac.kr, sylee@oslab.khu.ac.kr
}

\begin{abstract}
The model of the hybrid neural network is considered. This model consists of model ART-2 for clustering and perceptron for preprocessing of images. The perceptron provides invariant recognition of objects. This model can be used in mobile robots for recognition of new objects or scenes in sight the robot during his movement.
\end{abstract}

\section{Introduction}

Application of model Adaptive Resonance Theory (ART) of Grossberg-Carpenter [1] (in particular ART-2) is rather attractive to problem of solving of classification and clustering, because this model combines in itself properties of plasticity and stability, and also it does not demand a priori knowledge of the fixed quantity of necessary classes. So many different modifications of this model and its combinations with other neural networks are known $[2,3,4,5]$. But practically all of them are supervised learning and demand of teacher. Thus one of sufficient feature of model ART was refused.

In this paper we suggest one of hybrid model of neural network based on ART-2 and multi layer perceptron with error back propagation training algorithm (MLP-ART). In this model we try to keep the unsupervised learning and remove one sufficient disadvantage of ART.

Model ART-2 assumes usage of only one layer of neurons (not including entry, associated with sensors). It results to that the neural network works only with the metrics of primary features and calculates distance between images (for classification or creation of a new cluster - output neuron), usually using Euclidean distance. The absence of adapting to transformations of input vector to same one in real time is a result to that for real applications the model ART-2 is almost never used. For example, for clustering and pattern recognition by the mobile robot [6] it is required to recognize the object in different foreshortenings and allocating in different parts of a field of vision, i.e. recognition should be invariant concerning conversions of the map, such as shifts, rotations and others.

Multi layer perceptrons provide invariancy of recognition because in them hidden layers form secondary features during learning. Could say that in perceptrons each layer provides conversion of any feature space to another one.

${ }^{1}$ This work was supported by MIC Korea (IITA Visiting Professorship Program), Dr. S.Y.Lee is the corresponding author. 
In this paper the hybrid model is offered. It combines advantages of multilayer perceptron with learning by error back propagation [7] and of model ART-2. At first this paradigm was proposed in [8]. In this paper this paradigm and algorithms of model suffer sufficient improvements and continue developments, new experiments and conclusions are adduced.

\section{Main concepts and learning algorithm of hybrid model of neural network}

In suggested model the first some layers of neurons are organized as perceptron with forward connections. Its outputs are an inputs of model of Grossberg-Carpenter ART-2. Perceptron provides conversion of the metrics of primary features in the metrics of secondary features in space of considerably smaller dimension. Neural network ART-2 classifies images and uses secondary features to do it. Training of perceptron by error back propagation algorithm (EBP) provides "attraction" of an output vector of perceptron to centre of recognized cluster by ART-2. At that the weight vector of recognized cluster is desired output vector of multi layer perceptron. Could say, that the recognized class is a context in which system try to recognize other next images as same, and in some limits the system "is ready to recognize" its by this manner.

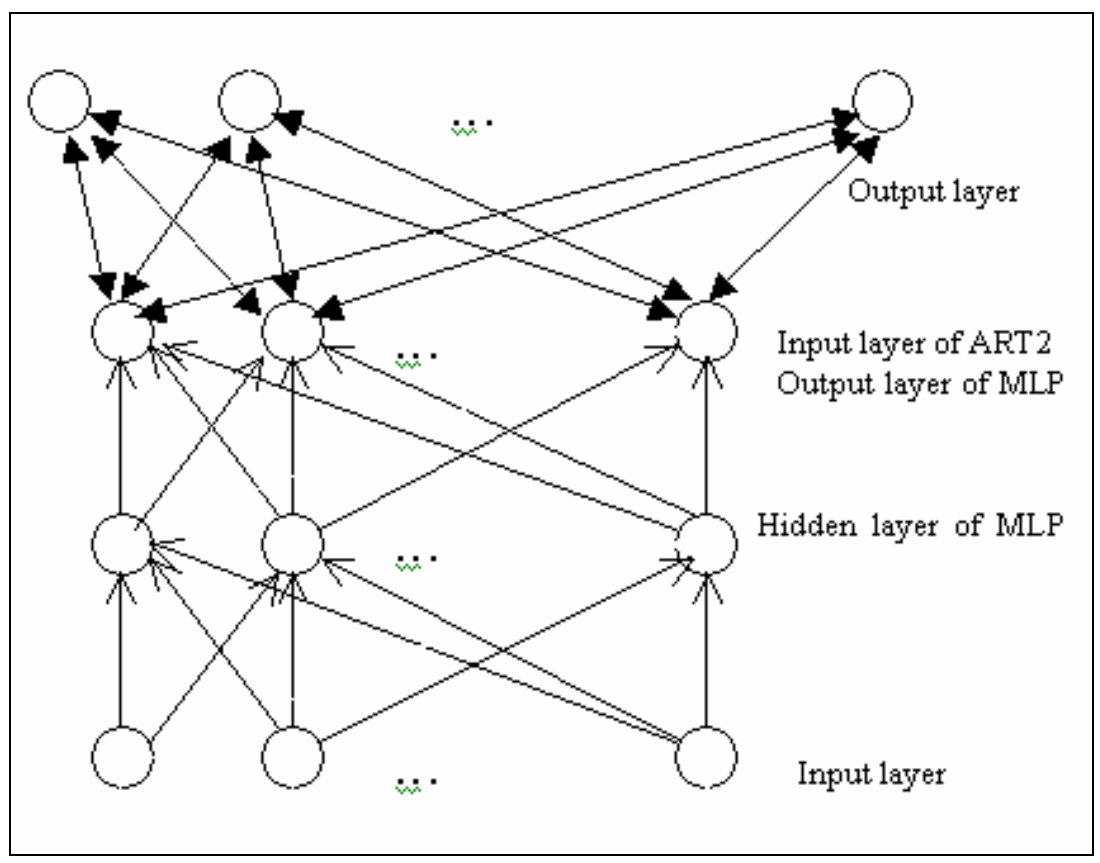

Fig. 1. Structure of hybrid neural network

Action of the suggested model is described by the following unsupervised learning algorithm: 
1. In the perceptron the weights of connections equal to half of quantity of neurons in the previous layer are formed. The quantity of output neurons $N_{\text {out }}$ of ART-2 is considered equal zero.

2. The next example is present to inputs. Outputs of perceptron are calculating.

3. If $N_{\text {out }}=0$, then the output neuron is formed with the weights of links equal to values of inputs of model ART-2 (the outputs of perceptron).

4. If $N_{\text {out }}>0$, in model ART-2 the algorithm of calculation of distances between its input vector and centers of existing clusters (the weight vectors of output neurons) is executing:

$$
d_{j}=\sqrt{\sum_{i}\left(x_{i}-w_{i j}\right)^{2}}
$$

where: $x_{i}-i^{\text {th }}$ digit of input vector of ART-2,

$$
w_{i j}-i^{\text {th }} \text { digit of weight of } j^{\text {th }} \text { output neuron (the cluster). }
$$

If the distance for the neuron-winner more than defined $R$ value (a vigilance or radius of cluster), the new cluster as well as in step 3 is formed.

5. If the distance for the neuron-winner is less $R$ in model ART-2 weights of connections for the neuron-winner are enumerating, approximating centre of a cluster to the input vector of model ART-2:

$$
w_{i m}=w_{i m}+\left(x_{i}-w_{i m}\right) /\left(1+N_{m}\right),
$$

where: $N_{m}$ - a number of recognized input vectors of $m^{\text {th }}$ cluster.

And for perceptron a recalculation of weights by algorithm "error back propagation" (EBP) are executing. Thus a new vector of weights of output neuron-winner of model ART-2 is viewed as output desirable vector for EBP, and the quantity of iterations can be little (in particular, there can be only one iteration).

6. The algorithm repeats from step 2 while there are learning examples in training set.

Action of the suggested model is explained in a figure 2 .

Here the space of secondary features in which by points are represented output vector of perceptron (input vector of model ART-2), centers of clusters are shown. In a figure the following points are represented:

1) - a new image for which the cluster with radius $R$ is created,

2 ) - the new image recognized as concerning to this cluster,

3 ) - the new centre of a cluster calculated in step 5 of algorithm,

4) - a new output vector of perceptron, approximated to centre of a cluster as a result of executing of algorithm "error back propagation",

$5)$ - the new image recognized as inhered to other cluster. 


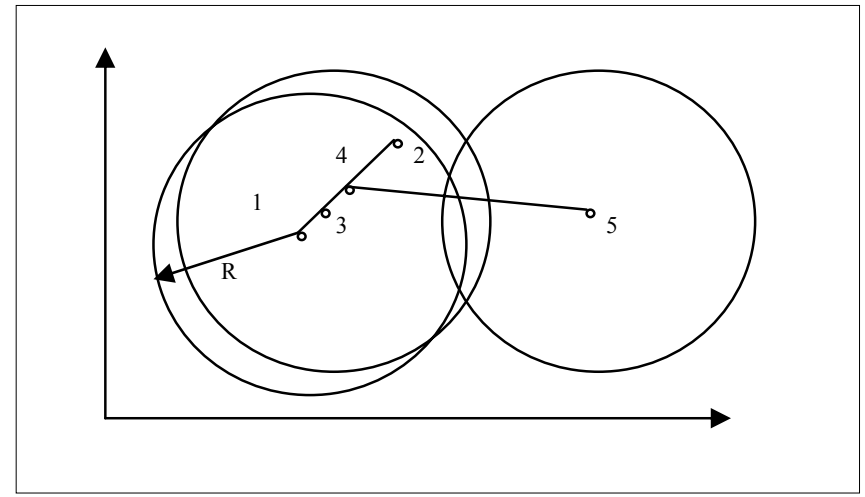

Fig. 2. Explanation of action of hybrid model

Earlier carried out experiments [8] with simple artificial images proved that a distance between a centroid of cluster and the input vector is increasing slowly during successful recognition of this cluster and its value depends on number of iterations of error back propagation algorithm.

\section{Experiments}

For research of the suggested model a framework for simulation of the neural network handling some sequences of patterns has been developed. As the sequence of images we used preliminary reduced to size $100 \times 100$ pixels shots from video. In a figure 3 three examples of images used in experiments are shown ( $1^{\text {st }}$ images of sequences $)$.

1)

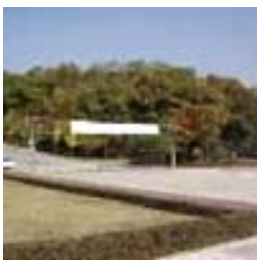

Fig. 3. The images (first Images from series) used in experiments

First series of images is sequence with moving cars, second - is moving bus in same place, third - moving images of chairs similar to pattern perceived by mobile robot in its moving into room.

The suggested algorithm of training without the teacher in experiments has shown good results at rather minor changing of each next example in training set. Thus the following parameters of model have been used:

Quantity of input neurons (pixels) - 10000 (100x100),

Quantity of neurons in hidden layer of perceptron - 20,

Quantity of output neurons of perceptron (in input layer of ART-2) $N_{\text {out }}-10$, 
Radius of cluster $R$ was used in experiments in different manners: 1) adapt and fix, 2) calculate for every image by formulas $S /\left(N_{a}{ }^{2}\right)$, where $\mathrm{S}$ - average input signal, $N_{a}-$ number of output neurons of perceptron, 3 ) calculated as $2 \mathrm{D}_{\min }$, where $\mathrm{D}_{\min }-$ minimal distance between input vector of ART2 and weight vectors in previous image.

Activation function of neurons of perceptron is rational sigmoid with parameter $\mathrm{a}=1$,

Value of learning step of perceptron is 1 ,

Number of iterations of recalculation of weights of perceptron is from 1 to 10 .

Some results of experiments are shown in figures 4 and 5.

From figure 4 one can see effect of partial forgetting. In repeated series of images system sometimes make mistakes and create new clusters. And at calculation of vigilance from last minimal distance effect of forgetting is more expressive. Moreover, in first present of series 2 the system makes mistakes in points 25 and 26 recognizing red bus as red car.

In figure 5 shown changing of distance between output vector of MLP (input vector of ART2) and centroid of recognized cluster at different number of iteration of EBP algorithm: 1, 3, 5, 7, 9. Dark line is corresponding to value 1 . One can see that distance decreases as result of action of EBP algorithm. Only in last point algorithm EBP already is unable to be opposed to strong changing of input vector. As result is creation of new cluster for next image.

Results of experiments show that:

1. Proposed model and learning algorithm may be used for processing of real visual images to detect novelty in series of images ignoring not enough strong difference between contiguous images.

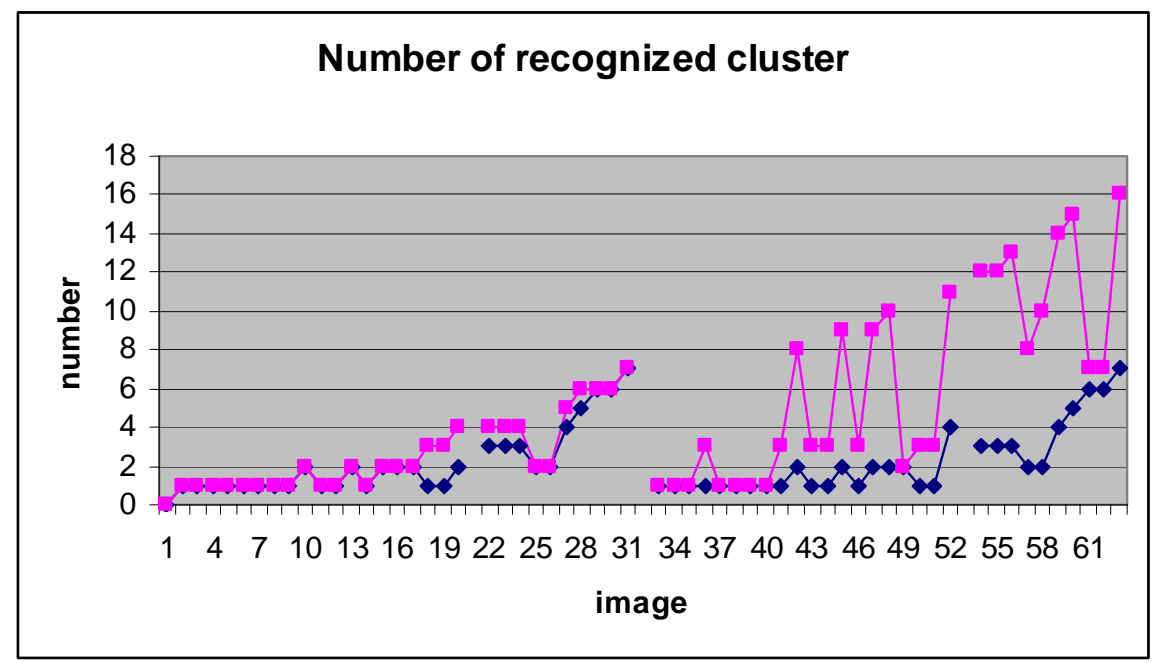

Fig. 4. For sequence of images of series 1, 2, 1, 2 (a dark points are corresponding to $2^{\text {nd }}$ kind of calculation of vigilance and light - to $1^{\text {st }}$ one). 


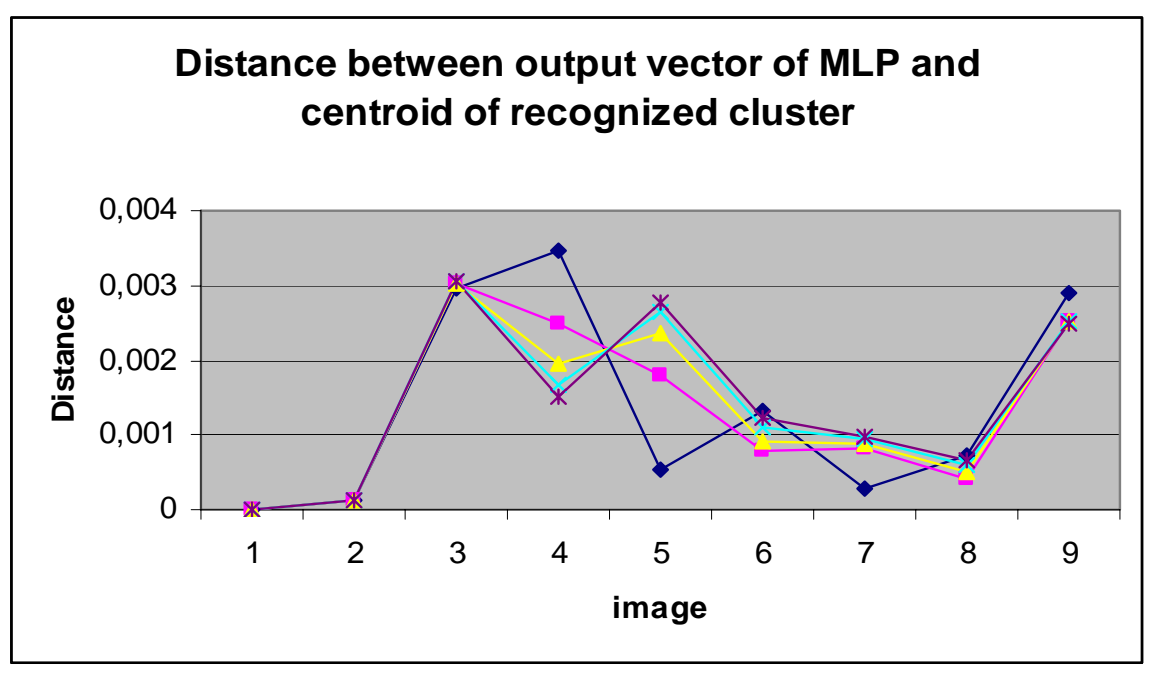

Fig. 5. For sequence of images of series 1 at different number of iteration of EBP algorithm:

$$
1,3,5,7,9 \text {. }
$$

2. May be to suppose that for every enough homogeneous series of images we can to select such value of vigilance which provides intuitively right behavior of this model.

3. At processing of strongly different series of images it is needed to calculate value of vigilance during learning (for every image). We propose for it empiric formulas $S /\left(N_{a}^{2}\right)$, where $\mathrm{S}$ - average input signal, $N_{a}$ - number of output neurons of perceptron. Binding of value of vigilance to last minimal distance between input vector and centroid of cluster proved to be worst.

4. Better results were obtained at prohibition of creation new cluster only for one image (after that system try to create also new cluster). In this case that new cluster is replaced by next new one. By this way the algorithm is oppose to strong increasing of number of clusters (output neurons) and to influence of random overshoots on keeping of structure of learned neural network.

\section{Conclusions}

The suggested hybrid model of the neural network can be used in the mobile robot when it is necessary to watch sequence of the images visible by the robot during its movement, and to extract in it new images (the objects), i.e. essential changes in the scene visible by the robot. More over, this model may be used in security systems for recognition of new objects or persons in sight of camera. Modification of this algorithm can be algorithm in which the quantity of created clusters is limited. In this case, if the quantity of clusters (output neurons) has achieved a limit, it is needed to decide what to do with images which are not recognized, i.e. which cannot be related to any existing cluster. In this case may be offered increasing of parameter R (radius of clusters) and to try to apply again algorithm of recognition and so until the new image will not be 
related to one of clusters. After that, it is necessary to reduce quantity of clusters (output neurons), to unify clusters with the centers which appear in one cluster, and to change weights of links between outputs of perceptron and outputs neurons-clusters.

Also one modification of this algorithm can be algorithm of training with the teacher in whom before to apply the procedure of increase of radius and decrease of quantity of output neurons, the system requests "teacher" what to do - to apply this procedure or to create a new cluster. As "teacher" there can be not only inquiry to user, but also any additional test for novelty of an image.

The following further researches of the suggested hybrid model of the neural network are planned:

- a mathematical substantiation of suggested algorithms,

- continue research of influence of perceptron and ART-2 parameters on results of action of the neural network,

- testing of the suggested model on program model of the mobile robot and the real robot.

\section{References}

[1] Carpenter, G. A., Grossberg, S.: Pattern Recognition by Self-Organizing Neural Networks, MIT Press, MA, Cambridge (1991).

[2] Shie-Jue Lee, Chun-Liang Hou.: An ART-Based Construction of RBF Networks. IEEE Trans. Neural Networks, 13(6) (2002) 1308-1321.

[3] Baxter, R.A.: Supervised Adaptive Resonance Networks. In: Proceedings of the Conference on Analysis of Neural Network Applications, ACM, VA, Fairfax (1991) 123-137.

[4] Jung-Hua Wang, Jen-Da Rau, Wen-Jeng Liu.: Two-Stage Clustering Via Neural Networks. IEEE Trans. Neural Networks, 14(3) (2003) 606-615.

[5] Hahn-Ming Lee, Chih-Ming Chen, Yung-Feng Lu.: A Self-Organizing HCMAC NeuralNetwork Classifier. IEEE Trans. Neural Networks, 14(1) (2003) 15-27.

[6] Gavrilov, A.V., Gubarev, V.V., Jo, K.-H., Lee, H.-H.: Hybrid Neural-Based Control System for Mobile Robot. In: Proceedings of $8^{\text {th }}$ Korea-Russia International Symposium on Science and Technology KORUS-2004, Vol. 1, TPU, Tomsk (2004) 31-35.

[7] Rumelhart. D.E.: Parallel Distributed Processing. In: Mcclelland J.L. (Eds.), Explorations in the Microstructure of Cognition, V.I, II, MIT Press, MA, Cambridge (1986).

[8] Gavrilov, A.V. Hybrid Neural Network Based on Models of Multi-Layer Perceptron and Adaptive Resonance Theory. In: Proceedings of 9th Korean-Russian International Symposium on Science and Technology KORUS-2005, NSTU, Novosibirsk (2005) 119122. 